\title{
Quantitative Characterisation of Surface and Interface Chemistry with X-ray Photoelectron Spectroscopy (XPS)
}

\author{
T. S. Nunney*, O. Mustonen* and R. G. White*
}

* Thermo Fisher Scientific, The Birches Industrial Estate, Imberhorne Lane, East Grinstead, West Sussex, RH19 1UB, UK

The chemistry and structure of material determines its interactions with other materials. In various applications ranging from biomedical devices to metals and sensors the composition of the surface is critical. The techniques are required to fully characterize the surface are often not present in the armoury of the microscopist. X-ray Photoelectron Spectroscopy (XPS) is a quantitative chemical analysis technique that probes only the outer few $\mathrm{nm}$ of the surface, enabling the analyst to characterize this important area of the material or device.

XPS, angle resolved XPS (ARXPS) and sputter profiling are methods that are ideally suited to the determination of the surface chemistry and the way in which that chemistry changes in the surface and near-surface region. XPS is the only analytical method providing quantitative elemental and chemical information with extremely high surface sensitivity and is ideal for comprehensively characterizing the elemental composition and chemical bonding states on surfaces and interfaces. For example, in the case of a low-emissivity coating on window glass, it is necessary to determine both that the structure of the stack conforms to expectation, and that there are no chemical irregularities. Typically samples such as these have a series of conducting and non-conducting layers ranging from $10 \mathrm{~nm}$ to $>100 \mathrm{~nm}$. XPS coupled with sputter profiling allows elucidation of the layer coating, as shown in figure 1. The capability of XPS to quantitatively assess the chemistry present is illustrated by the spectra in figure 2, which show how the Ni deposited around the important Ag layers acts as a sacrificial coating. The oxidized outer few $\mathrm{nm}$ are easily detected during the profile. In this presentation we will discuss how XPS can address a variety of surface problems in application areas as diverse as glass manufacture, mechanical lubrication, contact lens coating conformity, self-assembled monolayer development and wrapping paper design. 


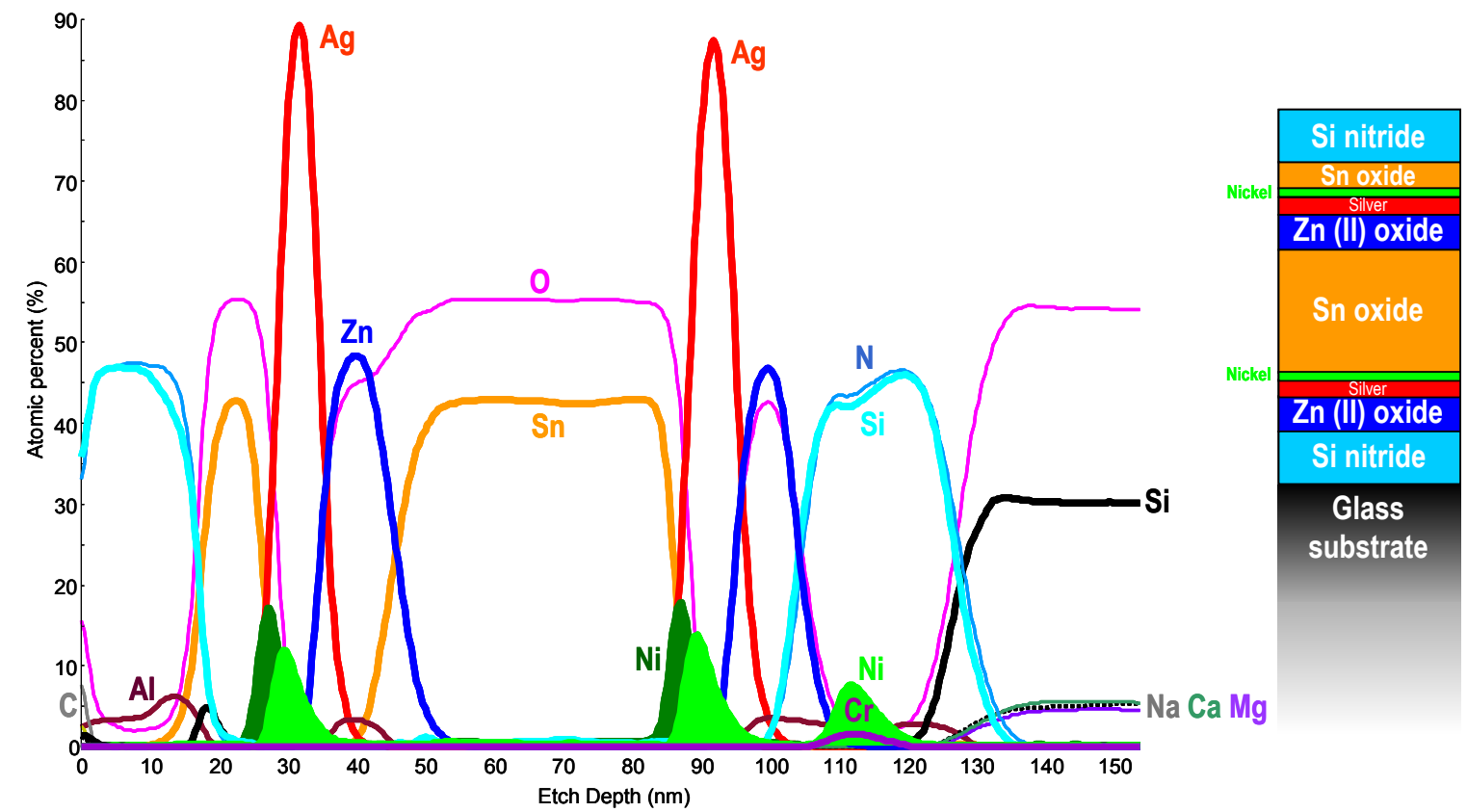

FIG. 1. XPS sputter depth profile of low-emissivity glass, illustrating both elemental and chemical information.

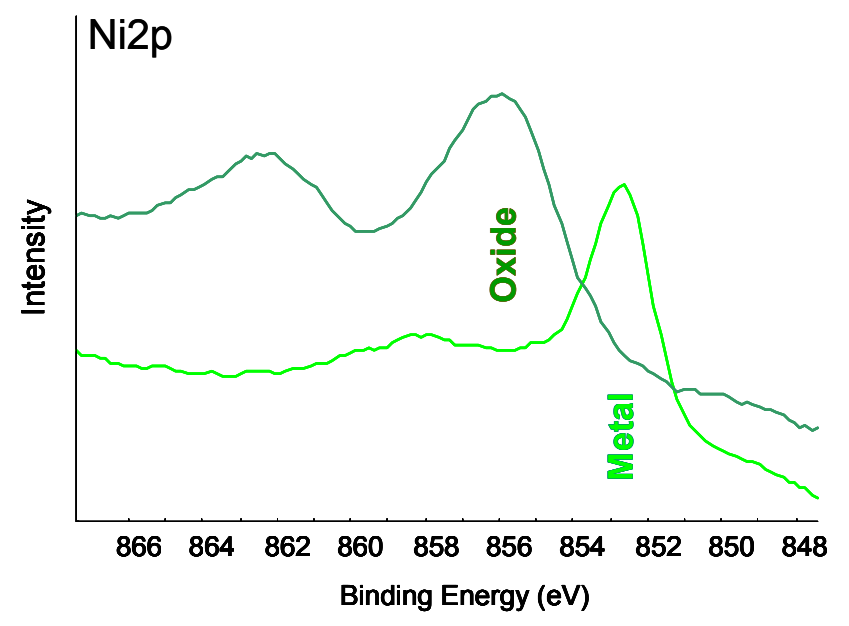

FIG. 2. Snapshot spectra showing the identification of the two chemical states of nickel present in the sample. 